\title{
LA GESTIÓN DEL CONOCIMIENTO Y LA EDUCACIÓN SUPERIOR UNIVERSITARIA
}

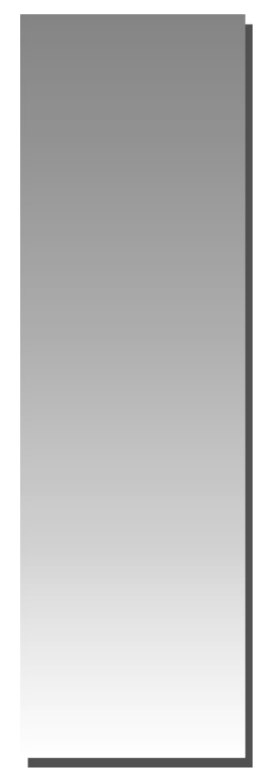

Teresa J. Ríos Delgado*

triosd@unmsmm.edu.pe

\section{RESUMEN}

La presente investigación se correlaciona la "gestión del conocimiento" y "la educación superior universitaria", con el objetivo de determinar la relación entre ambas. El tipo de investigación es descriptiva correlacional y explicativa. Es una investigación documental.

Como conclusión se determinó la relación entre ambos, lo que implica que la gestión del conocimiento influye significativamente en la educación superior universitaria en nuestra sociedad. Esto conlleva a que se deba establecer una planificación estratégica en el que la gestión del conocimiento sea una de las aristas más importantes en los diseños curriculares

Palabras clave: gestión del conocimiento, educación superior universitaria

\section{ABSTRACT}

This research has been developed in order to obtain a correlation between "knowledge management" and "university Education". This study is descriptive, correlational, explicative and a documental research. As conclusion the relationship between these two subjects was established therefore the knowledge management influences significantly at university education in our society

This drives us to determine a strategic planning based on knowledge management in curricula planning.

Keywords: knowledge management and university education.

* Doctora en Educación, Doctora en Gestión y Desarrollo, Magíster en Educación -Docencia en el Nivel Superior-. Traductora e intérprete. Bachiller en Derecho. Profesora auxiliar y docente investigadora. Docente de pregrado y posgrado en investigación. 


\section{INTRODUCCIÓN}

La gestión de conocimientos es un término surgido a principios de la década de los 90 , que ha adquirido mucha popularidad en los últimos años y que puede definirse como el conjunto de procesos que dirigen el análisis, diseminación, utilización y traspaso de experiencias, información y conocimientos entre todos los miembros de una organización para generar valor.

Se trata de "la tarea de desarrollar y explotar los recursos tangibles e intangibles del saber de una empresa". Los recursos tangibles serían las patentes, licencias, información sobre clientes, proveedores, productos y competidores, trabajos de investigación, etc. Los intangibles serían los conocimientos y experiencias de los empleados, el know-how de la empresa, etc.

Sin embargo, la gestión de conocimientos no consiste en el flujo indiscriminado de información a través de una organización, ya que la información es simplemente un conjunto de datos. Por el contrario, el conocimiento, el saber, implica depurar y añadir valor a la información, llegar a una solución o decisión en una situación real, interpretando esos datos dentro de un contexto.

Esta transferencia de conocimientos, de bestpractices, de know-hows... es lo que realmente aporta un valor diferencial a la empresa frente a sus competidores. Este tipo de gestión puede aportar grandes beneficios a una empresa, como reducción de costos, del tiempo de toma de decisiones, etc. En consideración a que la demanda social del conocimiento y su gestión con el objetivo de que el hombre pueda disponer de modelos de orientación en la situación que sean adecuados y oportunos para la toma de decisiones y la regulación de las acciones. En el desarrollo de la presente investigación, la educación superior universitaria, la demanda por educación superior puede aproximarse en términos de quienes, habiendo completado la secundaria, materializan su deseo de continuar estudios superiores mediante su postulación a alguna institución educativa de este nivel, o en términos de quienes, habiendo postulado, acceden a una vacante, es decir, de quienes ingresan. También es posible aproximar la demanda por educación superior en términos de quienes se encuentran cursando estudios superiores, es decir, analizando la matrícula, en tanto no todos los que ingresan a una instituciónde educación superior se matriculan en dicha institución.
Al mismo tiempo en que la demanda por educación universitaria aumentaba, durante los últimos veinte años, el número de universidades públicas y privadas también aumentó y no solo en las regiones en las que ya existía oferta de educación universitaria, sino también en regiones que no contaban con universidades. Con la excepciónde la Universidad Nacional Mayor de San Marcos, el porcentaje de postulantes que cada universidad recibe cada año tiende a disminuir en la medida en que aumenta la competencia ocasionada por la aparición de nuevas universidades.

Se puede apreciar que las demandas sociales constituyen un tipo de estimulación social del proceso de gestión del conocimiento, determinado por el carácter de las necesidades sociales y en los diferentes niveles de los problemas: global, regional, de la sociedad, institucional, sociopsicológico e individual. Entonces, ese conjunto de conocimientos del desarrollo de la ciencia y la tecnología, que garantizan la valoración adecuada de los elementos esenciales de una situación según sus requerimientos actuales y futuros, y posibilitan el esclarecimiento de las acciones a ejecutar, su dirección, regulación y corrección en función de los objetivos fijados son los que tienen fecha de caducidad, ya que el conocimiento científico es relativo, falible y provisorio.

De esta manera, en el proceso de desarrollo del conocimiento social es identificable, por otra parte, por el carácter de la necesidad de la sociedad, de la producción que sirve de estímulo al desarrollo del conocimiento el cual puede definirse, por ejemplo, por los problemas diarios que momentáneamente surgen en la comunicación, la frecuente avidez de conocimientos, las tareas prácticas de dirección y regulación de la conducta que aparecen episódicamente y una serie creciente de problemas en distintos niveles de organización social. Por otra parte, es factible distinguir un conjunto de conocimientos acumulados que posee determinada organización, es decir, determinado tipo de conocimientos predominantes.

Se puede afirmar que a cada tipo de estimulación social del conocimiento responde, en un proceso de contradicciones, determinado tipo de conocimiento. El cambio del tipo de estimulación social, por lo general se adelanta al del tipo de conocimiento. La transformación de los tipos de estimulación social y de los tipos de conocimientos forma una espiral del desarrollo, en la que 
se puede apreciar lo singular, lo particular y lo general en su relación dialéctica.

De esta manera, la gestión del conocimiento se relaciona con los contenidos que se transmiten y desarrollan en la universidad.

En base a lo anteriormente desarrollado, llegamos al siguiente problema de investigación:

¿En qué medida la gestión del conocimiento, como un componente de desarrollo social potencia la educación superior universitaria en el desarrollo de una sociedad?

Teniendo como objetivos: Analizar en qué medida la gestión del conocimiento, como un componente de desarrollo social potencia la educación superior universitaria en el desarrollo de una sociedad

La presente investigación se justifica en su aspecto práctico, ya que en el entorno actual de alta competitividad, globalización, desarrollo tecnológico, reducción de vida de los productos y de crisis mundial, resulta clave la gestión del conocimiento para lograr el éxito sostenible de la empresa. En este contexto, resulta realmente crítico desarrollar las habilidades en los estudiantes universitarios para adquirir información, transformarla en conocimiento, incorporarlo a la institución como aprendizaje, compartirlo rápidamente y ponerlo en práctica. Y la universidad pertenece a la sociedad y es su deber trabajar por su cambio. Es un deber ineludible que se lo impone la esencia misma de la institución. Por eso debe haber una defensa y la protección solidaria y recíproca entre la Universidad y la sociedad.

Esto es muy importante, pues existe una correlación entre gestión del conocimiento y la educación superior universitaria ya que gracias al conocimiento las universidades, gestionan la innovación, la creación de rutinas organizativas más eficaces, el crecimiento empresarial, la sostenibilidad, las capacidades adaptativas al entorno, etc. Todo ello proporciona productos más exitosos, excelentes profesionales y un nivel de calidad en la educación.

Se justifica en su aspecto teórico ya que la gestión del conocimiento es, por tanto, la dirección planificada y continua de procesos y actividades para potenciar el conocimiento e incrementar la competitividad a través del mejor uso y creación de recursos del conocimiento individual y colectivo.
La gestión del conocimiento tiene mucho que ver, entre otros factores, con los activos intangibles, con el aprendizaje organizacional, con el capital humano, intelectual y relacional.

Finalmente, se justifica en su aspecto metodológico, ya que cada vez que se pretende introducir un cambio en la cultura organizacional, existe una serie de factores que pueden suponer un obstáculo, y se hace más patente en el caso de la gestión del conocimiento por tratarse de una cuestión intangible. Se pueden encontrar diferencias importantes entre organizaciones tradicionales y maduras en el uso de la gestión del conocimiento.

\section{MÉTODOS}

Esta investigación sobre la relación entre "la gestión del conocimiento" y" la educación superior universitaria" en el desarrollo de una sociedad es una investigación empírica, porque es un hecho que ocurre en nuestra sociedad, y aplicada, porque se va enfrentar el problema proponiendo alternativas de solución.

El nivel de la investigación es explicativo porque buscamos conocer los factores causales que explican nuestro problema de investigación.

El diseño básico de esta investigación es no experimental-transeccional-explicativo correlacional. Es no experimental, debido a que no se harán pruebas; es decir, en esta investigación las variables no van a ser manipuladas. Es transeccional, porque nuestro objeto de estudio va a ser analizado en un periodo determinado de tiempo. Es explicativo correlacional, porque se trata de encontrar evidencias de cómo la variable independiente gestión del conocimiento potencia la educación superior universitaria.

\section{RESULTADOS}

El Perú está viviendo un etapa de oferta y demanda educativa, siendo el pico más alto la demanda de formación superior, que continuará los próximos años debido, en primer lugar, a la política adoptada por ciertos gobiernos que han iniciado una nueva modalidad de universidad: universidad-empresa, que tiene por objetivo no solamente aumentar el número de estudiantes de la enseñanza superior sino también en un lucro propio y, en segundo lugar, a las nuevas necesidades relacionadas con la educación y la formación permanente que se van perfilando, por ejemplo, 
la acreditación. Y esto va en contraparte con otros factores que desempeñan una función importante como, por ejemplo, la rigidez del mercado laboral o un espíritu empresarial menos desarrollado, que conllevan a su vez menos oportunidades de empleo en sectores innovadores.

La cooperación entre las universidades y el mundo industrial debe intensificarse y centrarse en la orientación hacia la innovación, la creación de nuevas empresas y, en términos más generales, la transferencia y difusión de los conocimientos.

La multiplicación de los lugares de producción de conocimientos. Ante la tendencia creciente de las empresas a subcontratar sus actividades de investigación con las mejores universidades, el contexto universitario resulta cada vez más competitivo.

Esta reorganización se plasma, por una parte, en la diversificación y la especialización cada vez mayores del saber y la aparición de campos de especialidades de investigación y enseñanza cada vez más específicos y precisos; por otro lado, el mundo académico necesita adaptarse urgentemente al carácter interdisciplinario de las cuestiones que plantean los grandes problemas de la sociedad, tales como el desarrollo sostenible, las nuevas enfermedades, la gestión de los riesgos, etc. Sin embargo, las actividades de las universidades, concretamente en lo que se refiere a la enseñanza, tienden a mantener su organización en función del sistema tradicional de disciplinas.

La universidad debe responder a las nuevas necesidades en materia de educación y formación que surgen con la economía y la sociedad del conocimiento y, especialmente, la necesidad cada vez mayor de enseñanza científica y técnica, de competencias transversales y de posibilidades de aprendizaje permanente que exigen una mayor permeabilidad entre los distintos elementos y niveles de los sistemas de enseñanza y formación.

En términos generales, las perspectivas de carrera profesional en nuestras universidades, caracterizadas por la multiplicidad de situaciones, son limitadas y bastante inciertas. No obstante, si bien los desafíos son numerosos, lo que hay en juego no es menos importante.

Muy aparte del factor económico, se debe mejorar la excelencia de las universidades en materia de investigación y enseñanza, es la única forma de lograr una mayor apertura de las universidades hacia el exterior y poder pensar en su atractivo a escala internacional.

\section{DISCUSIÓN}

La gestión del conocimiento en las instituciones de educación superior universitaria es un fenómeno del que se está tomando conciencia en forma reciente y del que aún no se puede hablar como un campo de conocimiento y práctica ya constituido. De ahí la relevancia de analizar en qué contexto y con qué propósitos se originan históricamente los elementos presentes en el proceso de su constitución y cómo se incorporan al ámbito educativo y a las organizaciones universitarias.

Podemos apreciar que hay acceso generalizado a las tecnologías deinformación y comunicación y por su relación con la economía del conocimiento. Es de esperar que la gestión del conocimiento constituya un factor determinante para reproducir y, quizás, para agudizar las diferencias e inequidades entre las universidades y sus modalidades, entre aquellas que logren incorporar procesos y tecnologías de gestión del conocimiento y las que no lo hagan.

Para entender la gestión del conocimiento en la universidad se propone adoptar una perspectiva epistemológica que considera al conocimiento como acción, en un modelo de aprendizaje organizacional, performance through learning, cuyo propósito central sea la mejora de los desempeños y el logro de resultados. Una perspectiva epistemológica en la que se reconozcan las dimensiones del conocer como representación "conocer qué" (know what) y del conocer como acción "conocer cómo" (know how), la cual es necesaria para fundamentar la distinción entre el saber acerca de y el saber en acción, en activo, situado e "incorporado"

\section{CONCLUSIONES}

1. Se comprueba que la gestión del conocimiento, como un componente del desarrollo social potencia satisfactoriamente la educación superior universitaria en el desarrollo de una sociedad.

2. Esto implica no solo un acercamiento en el desarrollo de los sistemas de la gestión del conocimiento, si no que nos encontramos ante un campo de múltiples facetas y perspectivas ya que la gestión del conocimiento es un fenómeno organizativo dinámico y continuo, constituido por diversos procesos con características y focos de interés variados. 
3. Igualmente, en el sentido que la influencia de la gestión del talento humano en la potenciación de la educación superior universitaria en el desarrollo de una sociedad es significativa, lo cual conlleva a que las tecnologías de información y comunicación (TIC) se pueden y se deben utilizar para extender la gestión del conocimiento más allá del ámbito tradicional del almacenaje, recuperación y distribución del conocimiento codificado y sistematizado. El estudiante universitario tiene las competencias para desarrollar y utilizar sus propias herramientas del conocimiento. Así mismo, el "know-how", como competencia cognitiva se relaciona directamente con la educación superior universitaria en el desarrollo de una sociedad. La gestión del conocimiento en educación está presente en otras obras sobre temáticas de la sociedad del conocimiento, de la sociedad del aprendizaje, o de la economía del conocimiento.

\section{LITERATURA CITADA}

BAÑEGIL PALACIOS, Tomás M. y SANGUINO GALVÁN, Ramón. (2004). Gestión del conocimiento y estrategia. Buenos Aires. Edit. Ariel

BURGHARDT MURGUÍA, Heidi C. y LOEWEN KLASSEN, Dieter W. (2002). Gerenciando una organización educativa hacia una organización que aprende - propuestas. Perú, Tesis para optar el Grado Académico de Magíster en Administración, Universidad del Pacífico.

CEPAL (2005) Políticas públicas para el desarrollo de sociedades de información en América Latina y el Caribe. Chile, Documento preparado por el División Desarrollo Productivo y Empresarial de la CEPAL, Publicación de las Naciones Unidas.

CHIAVENATO, Idalberto (2006). Introducción a la Teoría General de la Administración. Séptima edición, de McGraw-Hill Interamericana.

DALKIR, Kimiz (2005). Knowledge management in theory and practice. Estados Unidos, Elsevier/ Butterworth-Heinemann.

DAVENPORT, Thomas (2001). Working Knowledge: Cómo las organizaciones manejan lo que saben. Buenos Aires: Pearson Education.

ESTEBAN, Miguel A. (2005). La gestión del conocimiento en las Universidades.
GARCÍA-TAPIAL ARREGUI, Joaquín(2002). Gestión del conocimiento y empresa. España, EOI.

GÓMEZ VIEITES, Álvaro (2004). Sistemas de información: herramientas prácticas para la gestión empresarial. México, Alfaomega.

HUGOS, Michael (2003). Essentials of Supply Chain Management. John Wiley \& Sons, Ing. New Jersey, USA.

LEPELEY, María Teresa (2003) Gestión y Calidad en Educación. McGraw-Hill.

KUANG-TSAE, H., LEE, YANG, W. \& WANG, R..(2000). Calidad de la información y gestión del conocimiento. Madrid. Editorial AENOR.

MUÑOZ-SECA, BEATRIZY RIVEROLA, Josep (1997). Gestión del conocimiento. España, Biblioteca IESE de Gestión de Empresas, Universidad de Navarra, Ediciones Folio.

PÉREZ LINDO, Augusto y Otros (2005). Gestión del conocimiento. Grupo Editorial Norma. Buenos Aires.

RIVERO, S. (2002). Claves y pautas para comprender e implantar la gestión del conocimiento, un modelo de referencia. Fundación de la Escuela de Ingenieros de Bilbao. Socintec.

\section{REFERENCIAS VIRTUALES}

Carrión, J. (2001). "Nuevos modelos en Internet para gestionar el talento y el conocimiento".

Cinco Días. "Que aportan a la empresa las redes internas". Revista Emprendedores. En: http:// www.emprendedores.cl/articulos/mg32.htm

Chong, C. W. ; Holden, T.; Wilhelmij, P.; y Schmidt, R. (2000). "Where does knowledge management add value?". Journal of Intellectual Capital, Vol. 1, № 4, Pg. 366-380. En: http:// susanna.emeraldinsight.com/vl $=9622415 /$ $\mathrm{cl}=23 / \mathrm{nw}=1 / \mathrm{rpsv} / \sim 1118 / \mathrm{v} 2 \mathrm{n} 2 / \mathrm{s} 5 / \mathrm{p} 148$

La gestión del conocimiento (2011) Recuperado el 17 de julio 2012. Disponible en http://www. uoc.edu/dt/20251/index.html

Marcos, JM. (2000). "La gestión del conocimiento en correos y telégrafos". Club Intelect 2000. En: http://www.gestiondelconocimiento.com

Ortega, J.A. (2001). "Empleados formados e informados”. Expansión. 
Sarriegi, JM. (2000). "El conocimiento tácito: Lo que los ordenadores aún no pueden gestionar". Gestión del conocimiento.com. En: http://www.gestiondelconocimiento.com/ articulos_academicos.htm
Torres, S. (2001). "Gestión del conocimiento: la asignatura pendiente". Expansión, 22/ Mayo/2001.

Gestión del conocimiento.com. En: http://www. gestiondelconocimiento.com/articulos_ academicos.htm 